\title{
Minilaparoscopic cholecystectomy - the new non-visible scars technique. Preliminary report of first series
}

\author{
Tadeusz M. Wróblewski ${ }^{1}$, Konrad Kobryń ${ }^{1}$, Łukasz Nazarewski ${ }^{1}$, Marta Dec $^{2}$, Bogna Ziarkiewicz-Wróblewska ${ }^{3}$, \\ Marek Krawczyk ${ }^{1}$ \\ ${ }^{1}$ Department of General, Transplant and Liver Surgery, Medical University of Warsaw, Warsaw, Poland \\ ${ }^{2}$ Department of Anaesthesiology, Medical University of Warsaw, Warsaw, Poland \\ ${ }^{3}$ Department of Pathology, Medical University of Warsaw, Warsaw, Poland
}

Videosurgery Miniinv 2015; 10 (2): 150-154

DOI: 10.5114/wiitm.2015.52706

\begin{abstract}
Introduction: Surgery without scars is the dream of many patients and surgeons as well. It includes many new laparoscopic techniques (LESS, SILS, hybrid NOTES), but data concerning common bile duct (CBD) lesions are unavailable. Aim: To establish the new technique of minilaparoscopic cholecystectomy (MCh): non-visible scar intervention without increasing the risk of $C B D$ lesions.

Material and methods: Forty consecutive patients with symptomatic gallbladder lithiasis were qualified for elective laparoscopic cholecystectomy (LCh) using one $10 / 11 \mathrm{~mm}$ umbilical port, one $5 \mathrm{~mm}$ right suprapubic port and two minilaparoscopic, disposable, no-port graspers. There were 26 women and 14 men, with the mean age 56 (17-72) years and with the average body mass index 28 (18-33) kg/m $\mathrm{m}^{2} \mathrm{CO}_{2}$ Veress $15 \mathrm{~mm} \mathrm{Hg}$ pneumoperitoneum was performed after transumbilical incision, and the first 10/11 mm port was inserted at the beginning for the $5 \mathrm{~mm}$ laparoscope and finally for typical instruments. Next under camera control, a $5 \mathrm{~mm}$ trocar was inserted in the right 'bikini line'. To this port the laparoscope was relocated from the umbilicus, and under its control two minilaparoscopic, disposable, non-port graspers were introduced after small, $2 \mathrm{~mm}$ skin incisions in the right anterior axillary line and in the right mesogastrium to catch the gallbladder. Next through the umbilical port, using typical instruments, the cystic duct and artery were dissected, clipped and cut. The gallbladder was removed through the umbilical port whole. Results: There was no conversion to open cholecystectomy. In 5 cases drainage of the gallbladder lodge was necessary through a $5 \mathrm{~mm}$ port in the right bikini line. The time of the intervention ranged from 90 min during the introduction of the new method to 50 min for the last procedures. No postoperative complications were observed, and all patients were discharged at the same time as after conventional LCh.

Conclusions: Two-port laparoscopic cholecystectomy performed with two minilaparoscopic no-port graspers does not increase the risk of CBD lesions. It provides an excellent cosmetic effect and is very convenient for the surgeon like typical LCh.
\end{abstract}

Key words: laparoscopy, minilaparoscopic cholecystectomy, scar-less surgery.

\section{Introduction}

Miniinvasive technique applied for laparoscopic cholecystectomy (LCh) spread worldwide very quick- ly due to it having an incomparably better postoperative course than open technique. One of the important advantages of laparoscopic surgery is also a much better cosmetic effect in comparison to open

\section{Address for correspondence}

Prof. Tadeusz M. Wróblewski MD, PhD, Department of General, Transplant and Liver Surgery, Medical University of Warsaw,

1 A Banacha St, 02-097 Warsaw, Poland, phone: +48 601678 844, e-mail: t.wroblewski@videosurgeryjournal.com 
methods. So, still new modifications of typical laparoscopic techniques have been being developed recently, including the concept of single umbilical incision (SILS, LESS and others) and natural orifice transluminal endoscopic surgery (NOTES), to create no-visible-scar surgery [1-3].

At the beginning of LCh the main problem was to diminish iatrogenic biliary lesions. It was achieved thanks to widespread development of many training systems in laparoscopic surgery. Finally the percentage of biliary lesions was established for the last years and ranged between $0.1 \%$ and $0.3 \%$ but unfortunately was always higher than in open cholecystectomy.

Introduction of new techniques of LCh and application of new devices to these interventions requires quite new assessment of them, particularly the safe dissection of Calot's triangle structures [4]. However, there are no data concerning common bile duct (CBD) lesions during single port surgery or NOTES cholecystectomy yet.

\section{Aim}

In this report we present the technique of minilaparoscopic cholecystectomy (MCh) using minilaparoscopic, no-port graspers, which we developed to secure safety during this procedure, especially to avoid biliary tract lesions.

\section{Material and methods}

Forty consecutive patients (26 women, 14 men, aged 18-74 years) with symptomatic gallbladder cholelithiasis were qualified electively for MCh. Patients with suspected CBD stones were excluded from the study.

Average body mass index (BMI) was 28 (range: 18-33) $\mathrm{kg} / \mathrm{m}^{2}$, and in the study protocol patients with $\mathrm{BMI}>35 \mathrm{~kg} / \mathrm{m}^{2}$ were excluded from the study. The mean age of patients was 55 years (range: 17-72 years). The perioperative analgesic regimen consisted of paracetamol $1 \mathrm{~g}$ administered intravenously before induction of anesthesia and ketoprofen $100 \mathrm{mg}$ given approximately $20 \mathrm{~min}$ before emergence from anesthesia. In addition, at the end of the operation before wound closure all sites of trocar insertion were infiltrated with $0.25 \%$ bupivacaine $(14 \mathrm{ml}$ total: $8 \mathrm{ml}$ umbilicus, $2 \mathrm{ml}$ per each of the other three incisions). During the first $24 \mathrm{~h}$ parenteral analgesia was continued according to a standard protocol with paracetamol $1 \mathrm{~g}$ per $6 \mathrm{~h}$ and ketoprofen $100 \mathrm{mg}$ per $12 \mathrm{~h}$.

\section{Surgical technique}

All patients were placed on the operating table slightly on the left side $\left(20^{\circ}\right)$ in a $30^{\circ}$ reverse Trendelenburg position. The surgeon stood between the open patient's legs and the assistant at the right side of the patient.

After a $2 \mathrm{~cm}$ long transumbilical skin incision, $15 \mathrm{~mm} \mathrm{Hg}$ pneumoperitoneum was created with a Veress needle. First two typical $5 \mathrm{~mm}$ and 10/11 mm disposable trocars (Covidien, Norwalk, USA) for classical LCh were used. The first 10/11 mm trocar was introduced through the umbilical incision initially for the $5 \mathrm{~mm}$ laparoscope and finally for typical laparoscopic instruments. Next under $5 \mathrm{~mm}$ camera control, a $5 \mathrm{~mm}$ trocar was inserted in the right bikini line $3 \mathrm{~cm}$ to the right of the middle hypogastric line. To this port the laparoscope was relocated from the umbilicus and under its control the first disposable minilaparoscopic grasper (Stryker, USA) was inserted directly through the abdominal wall after small, pointed $2 \mathrm{~mm}$ skin incisions in the right middle abdomen. The second minilaparoscopic grasper was inserted similarly in the right anterior axillary line.

The position of these graspers, both to catch the gallbladder, were the same as in the typical, 4-trocar LCh. Using these graspers we can strongly and firmly catch the fundus and trunk of the gallbladder to open and expose the Calot triangle for dissection. Next through the umbilical port, using typical instruments, the cystic duct and artery were dissected, clipped and cut. The gallbladder was removed through the umbilical port whole.

When it was necessary to leave the drain in the gallbladder lodge, we moved it out through the $5 \mathrm{~mm}$ trocar in the right bikini line. Pneumoperitoneum desufflation through the ports was followed by closure of the fascia only in the umbilicus port site by 1-0 PDS (Ethicon, J\&J). The skin in the umbilicus was closed with nonabsorbable separated stitches, but the places after minilaparoscopic instruments and the $5 \mathrm{~mm}$ bikini line trocar were closed with steri-strips.

All patients obtained the same postoperative pain treatment assessed using a horizontal $10 \mathrm{~cm}$ visual analog scale (VAS) $1 \mathrm{~h}$ after the operation in the post-anesthetic care unit and further at postop- 
erative hours 6, 12, 24 in the surgical ward. If the recorded VAS was $>4$, morphine $0.1 \mathrm{mg} / \mathrm{kg}$ s.c. was administered. To prevent nausea and vomiting, patients were given a prophylactic dose of ondansetron $4 \mathrm{mg}$ i.v. approximately half an hour before the end of intervention. If the patient complained of nausea or an emetic episode (vomiting or retching) occurred in the postoperative course, an additional dose of ondansetron $4 \mathrm{mg}$ i.v. was administered.

\section{Results}

In all selective patients LCh was performed successfully. However, in 5 (12.5\%) cases 1 minilaparoscopic device in the right anterior axillary line was replaced with a $5 \mathrm{~mm}$ port for stronger gripping of the inflamed gallbladder wall. In 8 (20\%) patients after dissection of the inflammatory gallbladder one drain into its lodge was necessary to leave for 1 day introduced through the $5 \mathrm{~mm}$ bikini line port. The time of the intervention ranged from $1.5 \mathrm{~h}$ during the introduction of the new method to $50 \mathrm{~min}$ for the last procedures. It was related to the learning curve of the course and grade of inflammation of the gallbladder confirmed by histopathological findings. The pathologist reported chronic cholecystitis in 22 patients, cholecystitis with associated cholesterolosis in 11, and exacerbation of the cholecystitis in 7 patients. Rinsing and suction of the abdominal cavity was necessary in 15 (25\%) patients.

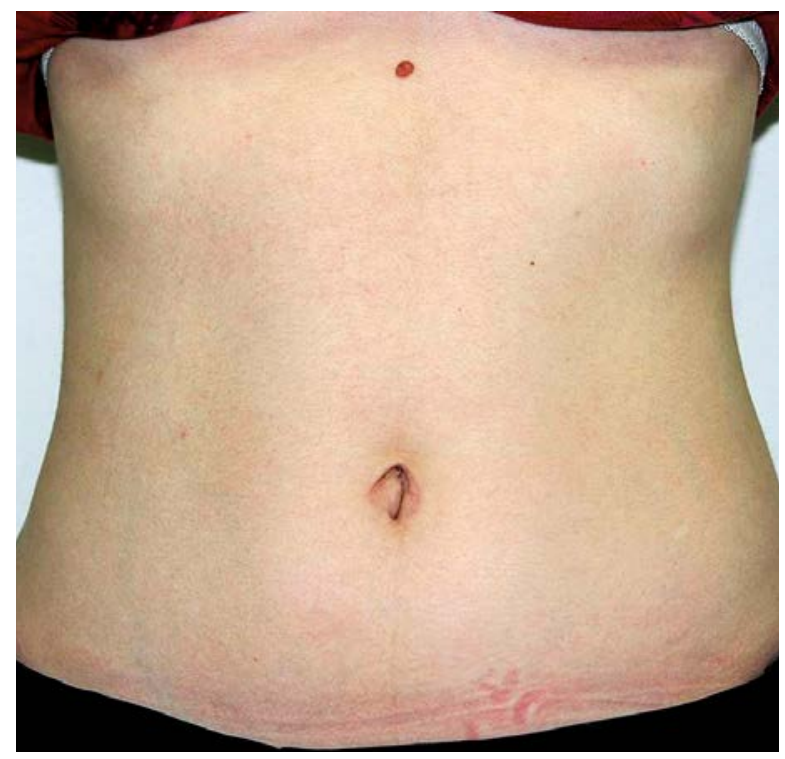

Photo 1. One month after mimilaparoscopic cholecystectomy
No postoperative complications were observed, and all patients were discharged on the second day after the intervention. The first outpatient follow-up was performed 10 days after the intervention when umbilical skin sutures were removed. After 1 month of observation there were no visible scars on the $a b$ domen, confirmed also after 2 months during the last outpatient follow-up (Photo 1). The patients' status was found to be similar as after conventional LCh during the postoperative period. The pathologist reported chronic cholecystitis in 22 patients, cholecystitis with associated cholesterolosis in 15, and exacerbation of the cholecystitis in 3 patients.

\section{Discussion}

Surgery without scars is the dream of many patients as well as surgeons. Nowadays, thanks to the involvement of many surgeons, engineers and equipment manufacturers, great progress in the development of surgical instruments that resulted in the creation of new surgical techniques has been achieved. Non-visible scar surgery is nowadays the new trend in surgery. Laparoscopic surgery is here the best area because of small incisions for trocars which are relatively easy to hide. The main idea of surgeons and medical constructors was to reduce the diameter of instruments or diminish the number of skin incisions [4]. As a result, many interesting devices such as special umbilical ports (SILS, LESS etc.) and curved surgical instruments were constructed and introduced into practice. Endoscopic companies in cooperation with endoscopists and laparoscopic surgeons have made a lot of prototypes of devices for new, rapidly developing techniques, e.g. NOTES, but this technique is still not ready for common use.

Diminished surgical injury and less invasive technique, but at the same time maximally hidden scars, were for all modern surgeons major goals in applying new methods and new technologies. However, among all goals, safety is the most important. Laparoscopic cholecystectomy was one of the first procedures for which the new trends were applied. Looking back at the history of LCh, many CBD lesions were reported in the first years of the introduction of this method, which systematically declined to stabilize at the level of $0.1-03 \%$. Finally, single incision surgery methods were vigorously developed, but until now we do not have any multicenter data concerning the results of cholecystec- 
tomy. The same also concerns NOTES and hybrid NOTES techniques [5].

We have not found any papers concerning operating comfort of surgeons performing these procedures. It means easy technical access to the operating area, which directly influences the procedure safety. The fight for every millimetre of space between the umbilical trocars and the place for the surgeon's hands manipulating the instruments in single port technique is very difficult for many surgeons if we compare it to well-established traditional LCh. Single site cholecystectomy did not give us an optimal operating position for hands and intraoperative comfort. Operative safety in my opinion was decreased by $10-15 \%$ compared to classic three- or four-port LCh. These are, of course, my personal feelings, not possible to measure objectively. That is why, finally, after single access or double incision LCh $[6,7]$ we decided to introduce minilaparoscopic cholecystectomy.

In this technique we use 2 minilaparoscopic disposable graspers, which are inserted through small point incisions (like for a Veress needle) in places typical for LCh in the right middle abdomen to catch the gallbladder very securely. Thanks that, we could easily open Calot's triangle for dissection. The standard 10/11 mm trocar in the umbilicus allowed us to use the typical laparoscopic instruments such as the hook, dissector, clip applier and scissors and give the surgeon a very good position for safe dissection of the cystic duct and artery.

The laparoscope in this technique was introduced at the beginning of the procedure through the umbilical port and was next translocated to the $5 \mathrm{~mm}$ bikini line port, and visibility during cholecystectomy was excellent and convenient for surgeons. The transfer of the camera from the umbilical port site to the suprapubic right bikini line provides much more space for instruments in this region than described by authors for all single access techniques $[1,2,4]$. Furthermore, the small $2 \mathrm{~mm}$ skin incisions for noport instruments and after the $5 \mathrm{~mm}$ trocar did not require stitching, but instead strips were applied. Separate stitches were only used for umbilical skin incision closure. Even in the case of drain placement through $5 \mathrm{~mm}$ port placement in the bikini line, we applied strips on wound closure after its removal on the second day.

Minilaparoscopic cholecystectomy is laparoscopic cholecystectomy with two port and two non-port minilaparoscopic graspers in order to achieve the best cosmetic effect. The described procedure is easy to learn, and in the case of technical problems additional ports can be applied (as in typical LCh). The idea of MCH using minilaparoscopic ports was conceived because of the lowering of the security of CBD exposure in the newly developed techniques of $\mathrm{LCh}$. The main goals of this procedure have been achieved: firstly to maintain excellent exposure of Calot's triangle elements during dissection and avoid CBD lesions, and secondly improvement of operative surgeons' comfort during intervention in comparison to other newly introduced techniques. This technique should be used in all specific indications for elective patients, but in cases with CBD stones resolved at the same time during laparoscopic cholecystectomy using minilaparoscopic instruments seems to be highly controversial $[8,9]$.

\section{Conclusions}

Two-port laparoscopic cholecystectomy performed with two minilaparoscopic no-port graspers does not increase the risk of $\mathrm{CBD}$ lesions. It provides an excellent cosmetic effect and is very convenient for surgeons like typical LCh, but further multicenter studies are needed.

\section{Conflict of interest}

The authors declare no conflict of interest.

\section{References}

1. Tacchino R, Greco F, Matera D. Single-incision laparoscopic cholecystectomy: surgery without a visible scar. Surg Endosc 2009; 23: 896-9.

2. Hodgett SE, Hernandez JM, Morton CA, et al. Laparoendoscopic single site (LESS) cholecystectomy. J Gastrointest Surg 2009; 13: 188-92.

3. Chamberlain RS, Sakpal SV. A Comprehensive review of single-incision laparoscopic surgery (SILS) and natural orifice transluminal endoscopic surgery (NOTES) techniques for cholecystectomy. J Gastrointest Surg 2009; 13: 1733-40.

4. Elazary R, Khalaileh A, Zamir G, et al. Single-trocar cholecystectomy using a flexible endoscope and articulating laparoscopic instruments: a bridge to NOTES or the final form? Surg Endosc 2009; 23: 969-72.

5. Suo G, Xu A. Clipless minilaparoscopic cholecystectomy: a study of 1,096 cases. J Laparoendosc Adv Surg Tech A 2013; 23 : 849-54.

6. Carvalho GL, Cavazzola LT, Rao P. Minilaparoscopic surgery not just a pretty face! What can be found beyond the esthetics reasons? J Laparoendosc Adv Surg Tech A 2013; 23: 710-3. 
7. Langwieler TE, Nimmesgern T, Back M. Single-port access in laparoscopic cholecystectomy. Surg Endosc 2009; 23: 1138-41.

8. Wróblewski TM, Piotrowicz S, Ziarkiewicz-Wróblewska B, Krawczyk M. Technique of double incision laparoscopic cholecystectomy (DILCH) as an alternative to SILS. Rev Portug Chir 2010; 12: 61-63.

9. Saad S, Strassel V, Sauerland S. Randomized clinical trial of single-port, minilaparoscopic and conventional laparoscopic cholecystectomy. Br J Surg 2013; 100: 339-49.

Received: 11.06.2015, accepted: 12.06.2015. 